\title{
Universality of Bias- and Temperature-induced Dephasing in Ballistic Electronic Interferometers
}

\author{
Y. Yamauchi ${ }^{1}$, M. Hashisaka ${ }^{1}$, S. Nakamura ${ }^{1}$, K. Chida ${ }^{1}$, S. Kasai ${ }^{1}$, T. Ono ${ }^{1}$, \\ R. Leturcq ${ }^{2}$, K. Ensslin ${ }^{3}$, D. C. Driscoll ${ }^{4}$, A. C. Gossard ${ }^{4}$, and K. Kobayashi ${ }^{1}$ \\ ${ }^{1}$ Institute for Chemical Research, Kyoto University, Uij, Kyoto 611-0011, Japan \\ ${ }^{2}$ IEMN - CNRS, Cité Scientifique, Avenue Poincaré BP 60069, Villeneuve d'Ascq, France \\ ${ }^{3}$ Solid State Physics Laboratory, ETH Zürich, CH-8093 Zürich, Switzerland and \\ ${ }^{4}$ Materials Department, University of California, Santa Barbara, California 93106, USA
}

(Dated: November 4, 2018)

\begin{abstract}
We performed a transport measurement in a ballistic Aharonov-Bohm ring and a Fabry-Pérottype interferometer. In both cases we found that the interference signal is reversed at a certain bias voltage and that the visibility decays exponentially as a function of temperature, being in a strong analogy with recent reports on the electronic Mach-Zehnder interferometers. By analyzing the data including those in the previous works, the energy scales that characterize the dephasing are found to be dominantly dependent on the interferometer size, implying the presence of a universal behavior in ballistic interferometers in both linear and non-linear transport regimes.

PACS numbers: 73.23.Ad, 71.10.Pm, 85.35.Ds, 03.65.Yz
\end{abstract}

Electron interference has been the central issue in mesoscopic physics since 1980's, which has been promoting our understanding on the phase and coherence of electrons in solids [1]. Recently, an electronic MachZehnder interferometer (MZI) [2] is invoking considerable interest for its possibility to create electron entanglement by two-particle interference [3, 4]. This MZI relies on the edge channel transport as it requires chirality of the electron beam, which is also an advantage as the coherence length of the edge electrons is expected to be long. Several experimental works on MZI [2, 5, , 6, , 7], however, reported that the interference visibility, a measure of coherence, decreases exponentially as the temperature increases. Furthermore, the experimentalists found $[5,7,7,8,9]$ the so-called "lobe structure", an unexpected phase reversal in the visibility at a certain bias voltage.

Several theoretical attempts $10,11,12,13$, have tried to explain the observed small energy scales that characterize the lobe structure as well as its drastic temperature dependence. The Coulomb interaction was proposed to be responsible for the observed energy scale proportional to the inverse of the size of MZI [11, 12, 13]. It is, however, still unclear whether or not the above observations are specific to MZI. Indeed, some theories [12, 13] suggest that similar effects may occur in other ballistic interferometers. To investigate this possibility constitutes the central motivation of the present experimental work.

Here we report the conductance measurements for an Aharonov-Bohm ring (ABR) around zero magnetic fields and for a Fabry-Pérot-type interferometer (FPI) in the integer quantum Hall (IQH) regime. In both cases, we observed the lobe-like structure and the exponential decay of the visibility with temperature, akin to those in MZI. By analyzing the present data as well as those obtained in previous works, the characteristic energy scales of the two effects are found to dominantly depend on the interferometer size.

Figure 1a shows the atomic force microscope (AFM) image of the ABR fabricated by local oxidation using an AFM 14] on a GaAs/AlGaAs heterostructure twodimensional electron gas (2DEG) (the electron density $3.7 \times 10^{11} \mathrm{~cm}^{-2}$ and the mobility $2.7 \times 10^{5} \mathrm{~cm}^{2} / \mathrm{Vs}$ ) [15]. Measurements were performed around zero magnetic field and in the IQH regime. The FPI [16, 17] was patterned by using electron beam lithography technique on an GaAs/AlGaAs heterostructure 2DEG (the electron density of $2.4 \times 10^{11} \mathrm{~cm}^{-2}$ and the mobility $2.7 \times$ $10^{5} \mathrm{~cm}^{2} / \mathrm{Vs}$ ). The scanning electron micrograph (SEM) image of the sample is shown in the inset of Fig. 2a. We tuned the magnetic field $(B)$ and the gate voltages so as to make a small interferometer in the IQH regime [16, 17]. For both samples, the two-terminal conductance as well as the differential conductance at finite dc source drain voltages $\left(V_{s d}\right)$ were measured by using lock-in techniques at $37 \mathrm{~Hz}$ with $5 \mu \mathrm{V}$ ac bias voltage. The samples were placed in a dilution refrigerator and the electron temperature is deduced from thermal noise measurement [18] with the lowest electron temperature $(T)$ of $125 \mathrm{mK}$.

First we show the result of the ABR below $50 \mathrm{mT}$. At zero bias voltage, a clear $\mathrm{AB}$ oscillation with a visibility (the ratio of the oscillatory to the non-oscillatory conductance components) larger than $8 \%$ at $125 \mathrm{mK}$ is observed (Fig. 1a). The period of the $\mathrm{AB}$ oscillation is $25 \mathrm{mT}$, being consistent with the lithographic ring geometry with a radius of $230 \mathrm{~nm}$ [15]. Figure $1 \mathrm{~b}$ presents the image plot of the conductance as a function of $B$ and $V_{s d}$. Importantly, a phase reversal of the oscillation is found to occur around $\left|V_{s d}\right| \sim 0.35 \mathrm{mV}$. In the upper panel of Fig. 1c the cross sections of Fig. 1b are shown at the lines denoted by "A" and "B", which correspond to the peak and dip of the $\mathrm{AB}$ oscillation at $V_{s d}=0 \mathrm{~V}$, 

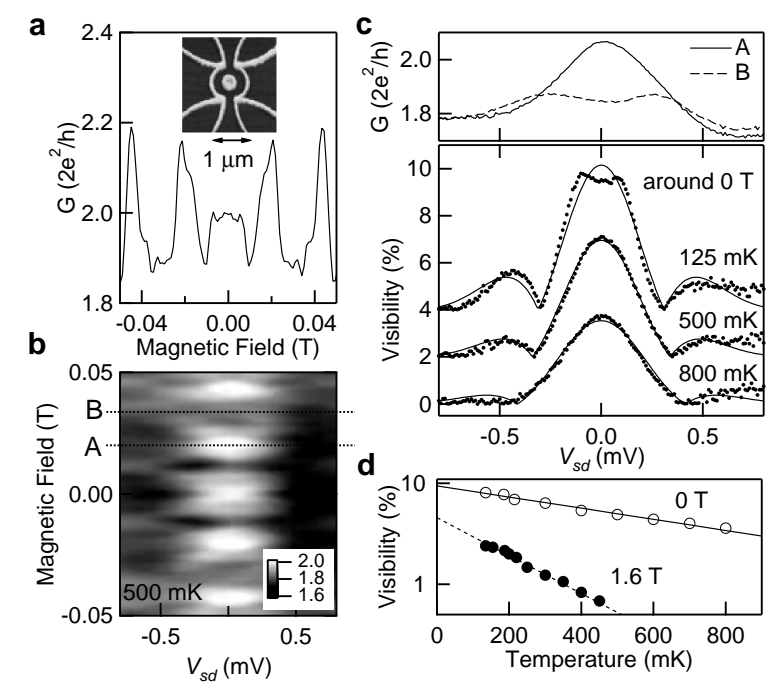

FIG. 1: (a) Conductance of the ABR at $125 \mathrm{mK}$ as a function of $B$. Inset: AFM image of the sample, white lines are oxidized area, under which the 2DEG is depleted [15]. (b) Image plot of the conductance as a function of $V_{s d}$ and $B$. The color scale is shown in units of $2 e^{2} / h$. (c) The upper panel shows the line profiles at the fixed magnetic field indicated by "A" and "B" in (b). The lower panel shows the corresponding visibility as a function of $V_{s d}$ at $T=125,500$, and $800 \mathrm{mK}$. The solid lines are the result of the fitting to Eqn. (1). They are vertical offset upward for clarity. (d) Electron temperature dependence of the zero-bias visibility for $0 \mathrm{~T}$ and $1.6 \mathrm{~T}$ (applied perpendicular to $2 \mathrm{DEG}$ ) plotted in a semi-logarithmic scale. The solid and dashed lines show the exponential decay function of $T$.

respectively. Because of the phase reversal of the oscillation, the resultant visibility $(\nu)$ presents a structure akin to the "lobe structure" known in MZI (see the bottom panel of Fig. 1c) [5, 7, 8, 9]. A similar phase reversal of the oscillation in the ABR was reported before [19], while no clear explanation has been given so far.

Figure 1c shows the visibility obtained at $T=125$, 500 , and $800 \mathrm{mK}$. The characteristic energy scale of the structure is estimated by using the following empirical equation as a function of $V_{s d}$ [7]:

$$
\nu=\nu_{0}\left|\cos \left(\frac{\pi e V_{s d}}{\epsilon_{L}}\right)\right| \exp \left(\frac{-\left(e V_{s d}\right)^{2}}{2 \epsilon_{0}^{2}}\right) .
$$

This is the product of the absolute value of a cosine curve, with period $\epsilon_{L}$, which characterizes the energy scale of the bias-induced phase reversal, and a Gaussian envelope with characteristic width $\epsilon_{0}$, which corresponds to the bias dephasing factor. $\nu_{0}$ is the zero-bias visibility. From the numerical fitting, $\epsilon_{0}$ and $\epsilon_{L}$ at $125 \mathrm{mK}$ are $0.30 \mathrm{meV}$ and $0.62 \mathrm{meV}$, respectively [20]. While the visibility rapidly decreases when the temperature changes from 125 to $800 \mathrm{mK}, \epsilon_{L}$ and $\epsilon_{0}$ are almost independent of temperature (Fig. 1c).

Although the characteristic energy scales are more than 10 times larger, these results look similar to those obtained for MZI [5, 7, 8, 9]. It is, however, important to examine here whether or not the phase reversal is explained by the magneto-electric $\mathrm{AB}$ effect [21] in the single particle picture. If the electrostatic potential difference between the two interfering paths in the ABR were responsible for the present observation, the bias dependence of the visibility would be determined by the term $\cos \left(e V_{s d} t_{0} / \hbar\right)$ [8, 21]. Here $t_{0}$ is the time for the electrons staying in the paths with different electrostatic potentials. In the present case, the condition to obtain $\epsilon_{L}=0.62 \mathrm{meV}$ yields $t_{0} \sim 3 \mathrm{ps}$ and therefore the electron traveling length, which would correspond to a path difference, is $0.7 \mu \mathrm{m}$ as the Fermi velocity of the present $2 \mathrm{DEG}$ is $2.5 \times 10^{5} \mathrm{~m} / \mathrm{s}$. However, such a path difference is unfeasible in the present symmetric geometry. Thus, the present lobe-like structure cannot simply be explained by the magneto-electric $\mathrm{AB}$ effect, which is also the case in MZI [8].

The analogy between the present result and those in MZI also lies in the fact that the zero-bias visibility has a clear exponential dependence on the electron temperature as shown in Fig. 1d. By fitting the temperature dependence to the function $\nu=\nu_{0} \exp \left(-T / T_{0}\right)$ we obtain $T_{0}=870 \pm 30 \mathrm{mK}$. The exponential decrease of the visibility with $T$ is consistent with previous reports for the ABR around $B=0 \mathrm{~T}[22,23]$. As the thermal broadening effect whose characteristic time scale is given by $\hbar / k_{B} T$ predicts $T_{0}=2.5 \mathrm{~K}$ for the size and the Fermi velocity of the ABR 23], the present dephasing is not explained by the thermal effect alone.

Next we discuss that a perfectly similar phenomenon occurs in the FPI. The conductance measurements for the FPI were performed in the IQH regime with a filling factor of 4 at $B \sim 2.26 \mathrm{~T}$. The gate voltages of the left and right pairs of the metallic gates in the SEM picture in Fig. 2a were fixed to $-1.1 \mathrm{~V}$ and $-1.0 \mathrm{~V}$, respectively, so as to tune the conductance of each point contact around $1.6 e^{2} / h$. The pair of the center gates were chosen to be around $-0.8 \mathrm{~V}$ to deplete the 2DEG underneath. By doing this, the one-dimensional state consisting of edge channels is formed along the area defined by the six gates, which is a small interferometer (see the upper right inset of Fig. 2a) [16, 17]. Figure 2a represents the oscillating component in the conductance through the FPI $\left(\Delta G_{A B}\right)$ as a function of $B$ or the center gate voltage. The $\mathrm{AB}$ period is $11.8 \mathrm{mT}$, indicating that the radius of the interferometer is around $350 \mathrm{~nm}$ [16] being consistent with the lithographic size of the device.

Figure $2 \mathrm{~b}$ shows the image plot of the conductance as a function of the gate voltage and $V_{s d}$. As in Fig. 1a, the present result shows the phase reversal of the oscillation at a finite bias voltage. From the cross section at the lines denoted "A" and "B" (Fig. 2b), the visibility is obtained as presented in Fig. 2c, which again looks similar to the lobe structure. Such a lobe-like structure is also obtained 

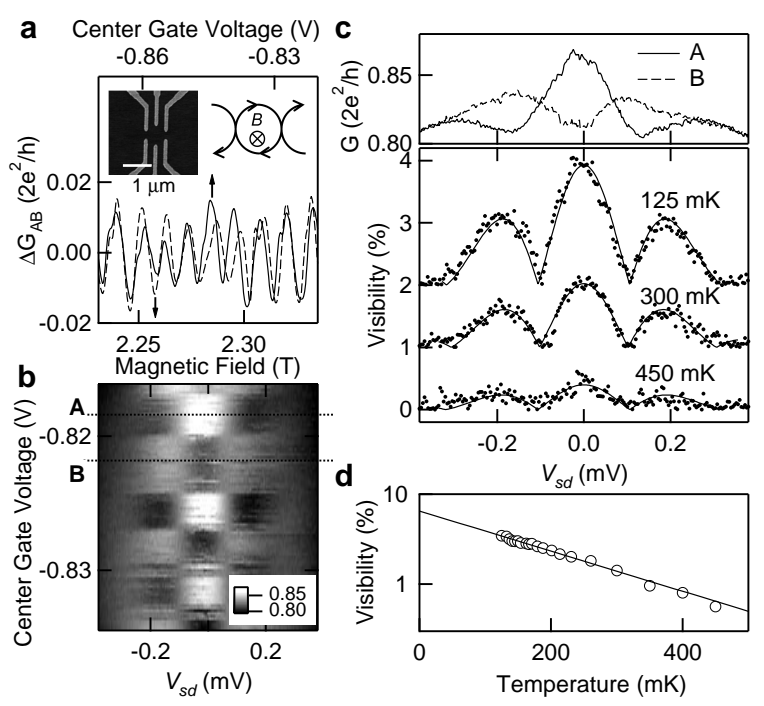

FIG. 2: (a) The oscillating components in the conductance of the FPI $\left(\Delta G_{A B}\right)$ as a function of $B$ (dashed line) and the center gate voltage (solid line). The insets show the SEM image of the sample in the left and the schematic drawing of the edge channels to construct the FPI in the right. (b) Image plot of the conductance as a function of $V_{s d}$ and the center gate voltage. The color scale is shown in units of $2 e^{2} / h$. (c) The upper panel shows the line profiles at the fixed gate voltages indicated by "A" and "B" in (b). The lower panel shows the corresponding visibility as a function of $V_{s d}$ at $T=125$, 300 , and $450 \mathrm{mK}$. The solid lines are the result of the fitting to Eqn. (1). They are vertically offset upward for clarity. (d) Electron temperature dependence of the zero-bias visibility is plotted in a semi-logarithmic scale. The line shows the exponential decay function of $T$.

from the differential conductance data as a function of $V_{s d}$ and $B$ instead of the gate voltage. The result of the temperature dependence of the lobe structure and the visibility at zero bias voltage between $125 \mathrm{mK}$ and $450 \mathrm{mK}$ are shown in Figs. 2c and 2d, respectively. Just as we did above, $\epsilon_{0}$ and $\epsilon_{L}$ are obtained to be $0.18 \mathrm{meV}$ and $0.21 \mathrm{meV}$, respectively. $T_{0} \sim 200 \mathrm{mK}$, which again cannot be explained by the thermal broadening effect.

Importantly, a completely similar phenomenon was observed in the $\mathrm{ABR}$ at $B \sim 1.6 \mathrm{~T}$ (filling factor 10). In this case, we obtain $\epsilon_{0}=0.25 \mathrm{meV}$ and $\epsilon_{L}=0.26 \mathrm{meV}$ with an $\mathrm{AB}$ period of $17 \mathrm{mT}$. The visibility also decreases exponentially with temperature with $T_{0}=240 \mathrm{mK}$ as shown in Fig. 1d. The different values of $\epsilon_{0}$ and $\epsilon_{L}$ compared to those at $B=0 \mathrm{~T}$ are due to a different interfering path geometry as indicated by the different $\mathrm{AB}$ period. However, it is noteworthy that $T_{0}$ is much reduced from the zero-field value, as will be discussed below.

Recently it was theoretically [12, 13] proposed that the lobe structure in MZI occurs through the Coulomb interaction; When the number of excess electrons present in MZI becomes two in the non-linear regime, the phases of the electrons are mixed due to the Coulomb interac-

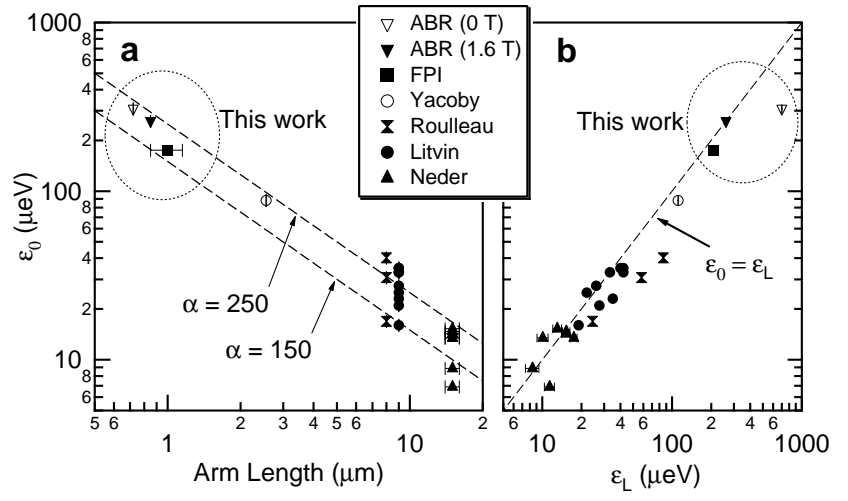

FIG. 3: (a) The energy scale $\left(\epsilon_{0}\right)$ of the lobe structure is plotted as a function of the arm length $(L)$ by using the data for the present $\mathrm{ABR}$ at $0 \mathrm{~T}$ and $1.6 \mathrm{~T}$, the present $\mathrm{FPI}$, the ABR at $0 \mathrm{~T}$ reported by Yacoby et al. [19], and MZI by Roulleau et al. [5, 9], Litvin et al. [7], and Neder et al. [8] Open points represent the data at zero magnetic field, and filled points in the IQH regime. The arm length of MZI by Neder et al. [8] is estimated from the SEM image of their sample. Different points with the same mark correspond to data obtained at different conditions of the magnetic field, the opening of the beam splitters, and so on. The two dashed lines indicate $\epsilon_{0}=\alpha / L$ with $\alpha=150$ and $250 \mu \mathrm{eV} \cdot \mu \mathrm{m}$. (b) The plot of $\epsilon_{L}$ vs $\epsilon_{0}$ shows that the two values are almost in the same range for a wide parameter range.

tion, resulting in the rapidly decreasing visibility with the phase reversal. Following their idea, a simple estimation of the energy scale for the present lobe-like structures can be performed for the ABR and the FPI as follows; The above effect occurs at $V_{s d}=V_{0}$ when the electron traveling time in one arm $L / v_{f}$ is almost twice the injection period $e / G\left|V_{0}\right|$ for the system with the conductance $G$. Here, $L$ is the arm length calculated by the oscillation period and $v_{f}$ is the Fermi velocity $\left(2.5 \times 10^{5} \mathrm{~m} / \mathrm{s}\right.$ for the ABR at $B=0 \mathrm{~T}$ ) and the edge velocity for the FPI (typically $3 \times 10^{4} \mathrm{~m} / \mathrm{s}$ ), respectively. The value $e V_{0}$ to characterize this effect is obtained to be $0.74 \mathrm{meV}$ for the $\mathrm{ABR}$ at $B=0 \mathrm{~T}\left(L=0.72 \mu \mathrm{m}\right.$ and $\left.G \sim 4 e^{2} / h\right)$ and to be $0.15 \mathrm{meV}$ for the FPI $\left(L=1.1 \mu \mathrm{m}\right.$ and $\left.G \sim 1.6 e^{2} / h\right)$. As these values are comparable to the observed $\epsilon_{0}$ or $\epsilon_{L}$ in each case, given the simplicity of the discussion, the dephasing due to the correlation between the excess electrons satisfactorily explains what happens in the nonlinear electronic interferometer.

Having proven that the above discussion essentially captures the dephasing in the ABR and FPI in the nonlinear regime, it is instructive to compare the present results with previous reports on ballistic interferometers to check the universality. The dependence of $\epsilon_{0}$ on the length of the arm of the interferometer $(L)$ (the averaged length between the two arms) is compiled in Fig. 3a. We plotted the data obtained for the present ABR at $0 \mathrm{~T}$ and $1.6 \mathrm{~T}$, the present FPI, the $\mathrm{ABR}$ at $0 \mathrm{~T}$ [19], 


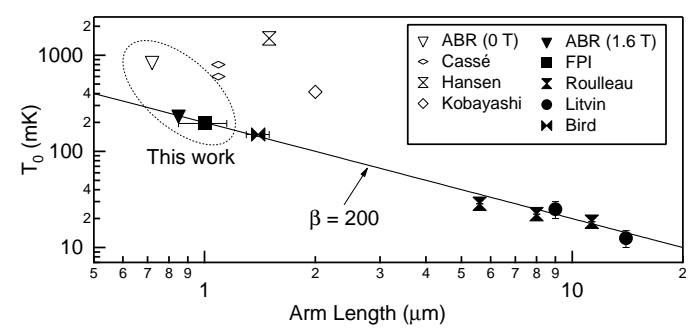

FIG. 4: The dependence of $T_{0}$ on the arm length $(L)$ of the interferometers is plotted by using the data obtained for the present $\mathrm{ABR}$ at $0 \mathrm{~T}$ and $1.6 \mathrm{~T}$, the present FPI, the FPI by Bird et al. [17], the ABR by Cassé et al. [24], Hansen et al. 22] and Kobayashi et al. 23] and MZI by Roulleau et al. [5, 9], Litvin et al. [7]. Filled points were those obtained under IQH regime, while opened points were at zero magnetic field.

and MZI [5, 7, 8, 9]. Interestingly, this graph shows that $\epsilon_{0}$ scales inversely proportional to the arm length, $\epsilon_{0}=\alpha / L$, where $\alpha$ falls between 150 and $250 \mu \mathrm{eV} \cdot \mu \mathrm{m}$ as shown in Fig. 3a. Fig. 3b shows the relation between $\epsilon_{0}$ and $\epsilon_{L}$, showing that $\epsilon_{0} \sim \epsilon_{L}$ holds for a wide range of the interferometer sizes. $\alpha$ is determined in principle in the same way as above by taking into account the Fermi (or group) velocity and the linear conductance. As theory suggests [12, 13], the variation of $\alpha$ in Fig. 3a and the slight deviation between $\epsilon_{0}$ and $\epsilon_{L}$ may be explained by including the effective Coulomb interaction in the interferometers with the realistic geometry. Also it is experimentally shown that $\epsilon_{0}$ and $\epsilon_{L}$ can be controlled by the opening of the beam splitter [9] or the magnetic field [7, 8], suggesting that the arm length is not the only parameter to affect the lobe structure. However, Fig. 3a clearly demonstrates that the relevant energy scale to describe the lobe structure in several kinds of interferometers is primarily determined by the size, which is the central result of the present experimental work.

We also found that $T_{0}$, which characterizes the dephasing in the linear regime, strongly depends on $L$ as shown in Fig. 4, where we compile the $T_{0}$ values obtained for the present $\mathrm{ABR}$ at $0 \mathrm{~T}$ and $1.6 \mathrm{~T}$, the present FPI, the FPI [17], the ABR [22, 23, 24] and MZI [5, 7, 9]. The plots indicate another universal behavior $T_{0}=\beta / L$ with $\beta=200 \mathrm{mK} \cdot \mu \mathrm{m}$ for the edge transport regime [27]. However, the data for the ABR at zero magnetic field strongly deviate from this line. This suggests that the difference of the group velocity between both is important and the coherence time but not the coherence length matters. The present notable difference between the zero-field state and the edge state may suggest that the environmental property in the vicinity of the interferometer is responsible for the temperature-induced dephasing [23, 25, 26].

To conclude, we found the "lobe structure" in the ballistic ABR and FPI, being analogous to those observed in MZI's. The characteristic energy scales are semi- quantitatively explained as due to the Coulomb interaction between the excess electrons in the interferometer. By compiling the energy scales for the lobe structure and the temperature dependence of the visibility, they are found to be proportional to the inverse of the interferometer size. The presence of such universality in dephasing, which is unveiled through the measurement on the present small electron interferometers, will shed new light on the coherence in ballistic systems.

We appreciate fruitful comments from H. W. Lee and A. Helzel. This work is supported by KAKENHI, Yamada Science Foundation, and Matsuo Science Foundation.

[1] Y. Imry, Introduction to Mesoscopic Physics(Oxford University Press, Oxford, 1997).

[2] Y. Ji et al., Nature (London) 422, 415 (2003).

[3] P. Samuelsson, E. V. Sukhorukov, and M. Büttiker, Phys. Rev. Lett. 92, 026805 (2004).

[4] I. Neder et al., Nature (London) 448, 333 (2007).

[5] P. Roulleau et al., Phys. Rev. Lett. 100, 126802 (2008).

[6] L. V. Litvin, H.-P. Tranitz, W. Wegscheider, and C. Strunk, Phys. Rev. B 75, 033315 (2007).

[7] L. V. Litvin et al., Phys. Rev. B 78, 075303 (2008).

[8] I. Neder et al., Phys. Rev. Lett. 96, 016804 (2006).

[9] P. Roulleau et al., Phys. Rev. B 76, 161309 (2007).

[10] J. T. Chalker, Y. Gefen, and M. Y. Veillette, Phys. Rev. B 76, 085320 (2007) and references therein.

[11] I. P. Levkivskyi and E. V. Sukhorukov, Phys. Rev. B 78, 045322 (2008).

[12] S. -C. Youn, H.-W. Lee, and H. -S. Sim Phys. Rev. Lett. 100, 196807 (2008).

[13] I. Neder and E. Ginossar Phys. Rev. Lett. 100, 196806 (2008).

[14] R. Held et al., Appl. Phys. Lett. 73, 262 (1998).

[15] R. Leturcq et al., Physica E 35, 327 (2006).

[16] B. J. van Wees, et al., Phys. Rev. Lett 62, 2523 (1989).

[17] J. P. Bird et al.,Phys. Rev. B 50, 14983 (1994).

[18] M. Hashisaka et al., Phys. Rev. B 78, 241303(R)(2008).

[19] A. Yacoby, R. Schuster, and M. Heiblum, Phys. Rev. B 53, 9583 (1996).

[20] The small dip around $V_{s d}=0 \mathrm{~V}$ in the $125 \mathrm{mK}$ data is due the mesoscopic fluctuation inside the ABR, which does not affect the present discussion on the characteristic energy scales. The dip disappears above $400 \mathrm{mK}$.

[21] W. G. van der Wiel et al., Phys. Rev. B 67, 033307 (2003).

[22] A. E. Hansen et al., Phys. Rev. B 64, 045327 (2001).

[23] K. Kobayashi, H. Aikawa, S. Katsumoto, and Y. Iye, J. Phys. Soc. Jpn 71, L2094 (2002).

[24] M.Cassé et al., Phys. Rev. B 62, 2624 (2000).

[25] G. Seelig, S. Pilgram, A. N. Jordan, and M. Büttiker, Phys. Rev. B 68, 161310R (2003).

[26] P. Roulleau et al., Phys. Rev. Lett. 101, 186803 (2008).

[27] Recently it was theoretically discussed that there is a universal relation between $\alpha$ and $\beta$ [1]. 